\title{
Neutrino mass hierarchy and Majorana CP phases within the Higgs Triplet Model at LHC.
}

\section{Julia Garayoa*}

IFIC (CSIC-Univ. Valencia)

E-mail: garayoa@ific.uv.es

\section{Thomas Schwetz}

CERN

E-mail: schwetz@cern.ch

In the Higgs Triplet Model neutrino masses are generated by the vacuum expectation value of an $S U(2)_{L}$ scalar triplet. We consider this model and we assume that the doubly charged component of the triplet has a mass in the range of several $100 \mathrm{GeV}$, such that it is accessible at LHC. Its decay into like-sign leptons provides a clean experimental signature, which allows for a direct test of the neutrino mass matrix. By exploring the branching ratios of this decay into leptons of various flavours, we explore the possibility of determining the type of neutrino mass hierarchy and measuring Majorana $\mathrm{CP}$ phases of the lepton mixing matrix.

10th International Workshop on Neutrino Factories, Super beams and Beta beams

June 30 - July 52008

Valencia, Spain

\footnotetext{
* Speaker.
} 


\section{Introduction}

We consider the Higgs Triplet Model, where neutrino masses are generated by the vacuum expectation value $v_{T}$ of a scalar $S U(2)_{L}$ triplet $\Delta\left(H^{ \pm \pm}, H^{ \pm}, H^{0}\right)[1]$ :

$$
\mathscr{L}_{\Delta}=f_{a b} L_{a}^{T} C^{-1} i \tau_{2} \Delta L_{b}+\text { h.c. } \rightarrow \frac{1}{2} v_{L a}^{T} C^{-1} M_{a b} v_{L b}+\text { h.c. } \quad \text { with } \quad M_{a b}=\sqrt{2} v_{T} f_{a b} .
$$

If the Higgs triplet is light enough, its doubly charged scalar component $H^{++}$could be produced at LHC via a Drell-Yan process [2]. The production cross section depends only on the mass of the scalar. For an integrated luminosity of $300 \mathrm{fb}^{-1}$, about $10^{3}$ scalar pairs would be produced for a mass around $300 \mathrm{GeV}$. These scalars can decay into same-sign leptons $H^{++} \rightarrow l_{a}^{+} l_{b}^{+}$. This decay provides a very spectacular signature, free from any Standard Model background. Moreover, the decay rate is proportional to the modulus of the corresponding element of the neutrino mass matrix:

$$
\mathrm{BR}_{a b} \equiv \mathrm{BR}\left(H^{++} \rightarrow \ell_{a}^{+} \ell_{b}^{+}\right) \equiv \frac{\Gamma\left(H^{++} \rightarrow \ell_{a}^{+} \ell_{b}^{+}\right)}{\sum_{c d} \Gamma\left(H^{++} \rightarrow \ell_{c}^{+} \ell_{d}^{+}\right)}=\frac{2}{\left(1+\delta_{a b}\right)} \frac{\left|M_{a b}\right|^{2}}{\sum_{c d}\left|M_{c d}\right|^{2}}
$$

This opens a very interesting link between neutrino physics and collider physics: seeing this kind of events at LHC would be a direct test of the neutrino mass matrix.

In addition to the lepton channel the doubly charged Higgs can in principle decay also into charged Higgses and/or the $W: H^{++} \rightarrow H^{+} H^{+}, H^{++} \rightarrow H^{+} W^{+}, H^{++} \rightarrow W^{+} W^{+}$. The first two decay modes depend on the mass splitting within the triplet, and we assume that they are kinematically suppressed. The branching ratio between $\ell^{+} \ell^{+}$and $W^{+} W^{+}$decays is controlled by the relative magnitude of the triplet Yukawas $f_{a b}$ and the vacuum expectation value $v_{T}$.

In this model contributions to lepton flavour violating processes, $g_{\mu}-2$, and to the electron electric dipole moment are expected. The strong experimental bounds on this type of processes put some constraints on the Yukawa couplings, the most stringent one coming from $\mu \rightarrow$ eee.

We can obtain an estimate for the interesting range of the yukawa couplings: $4 \times 10^{-7} \lesssim f_{a b} \lesssim$ $5 \times 10^{-4}$, for $M_{H^{++}}=100 \mathrm{GeV}$. The upper bound comes from the decay $\mu \rightarrow$ eee and the lower one from the requirement that $\Gamma\left(H^{++} \rightarrow W^{+} W^{+}\right) \lesssim \Gamma\left(H^{++} \rightarrow \ell_{a}^{+} \ell_{b}^{+}\right)$, together with the constraint from cosmology on neutrino masses, $v_{T} f_{a b} \lesssim 10^{-10} \mathrm{GeV}$.

Let us note that in the minimal version of this model the baryon asymmetry of the universe cannot be generated by leptogenesis, and one has to invoke some other mechanism beyond the model. However, in this case lepton number violating decays of the doubly charged scalar of the model might destroy the pre-generated baryon asymmetry. ${ }^{1}$ To avoid this to happen one has to require that these decays never come into equilibrium. This translates into a much stronger upper bound on the yukawas than the one given above: $2 \times 10^{-5} \lesssim f_{a b} \lesssim 5 \times 10^{-4}$, for $M_{H^{++}}=100 \mathrm{GeV}$.

\section{Numerical Analysis and Results}

In our analysis [3] we assume that a sufficient number of scalar pairs decaying into same sign leptons are observed. The decay $H^{++} \rightarrow l_{a}^{+} l_{b}^{+}$can occur in every flavour combination, including

\footnotetext{
${ }^{1}$ We thank Hitoshi Murayama for pointing out this problem to us.
} 


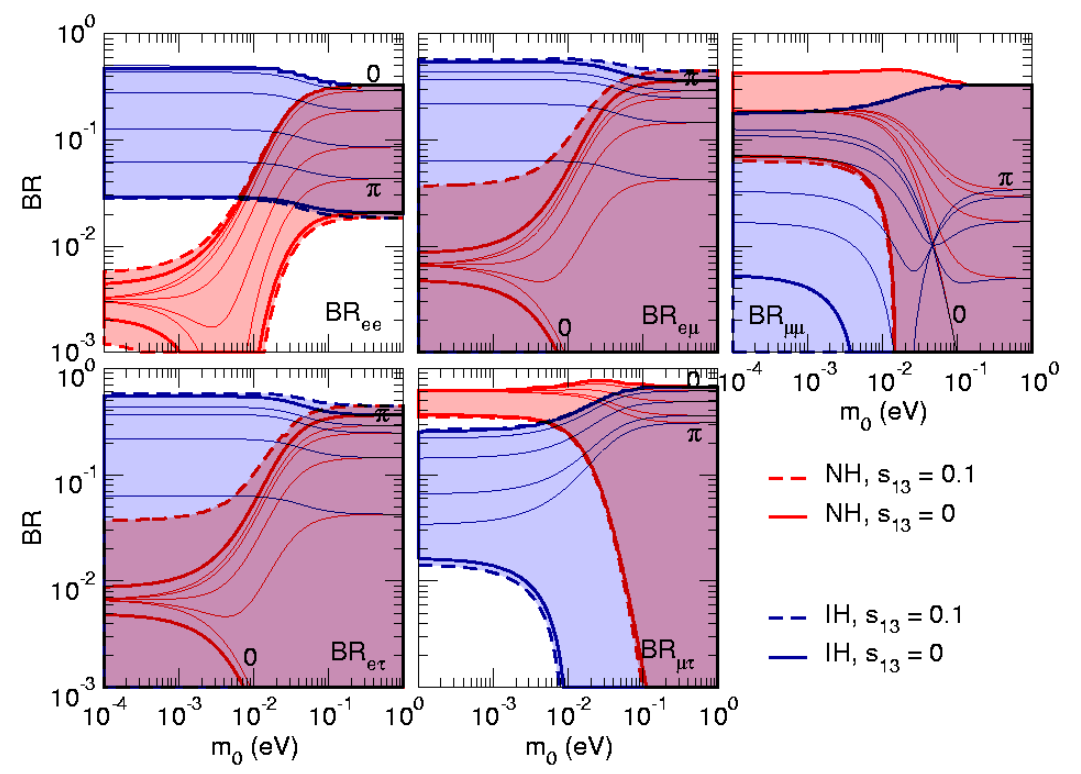

Figure 1: Branching ratios $\mathrm{BR}\left(H \rightarrow \ell_{a} \ell_{b}\right)$ as function of the lightest neutrino mass $m_{0}$ for NH (light-red) and IH (dark-blue). The thick solid lines are for $s_{13}=0$, and the thick dashed lines for $s_{13}=0.1$, where the dependence on phases as well as the uncertainty of solar and atmospheric oscillation parameters at $2 \sigma$ are included. The thin solid lines show the branchings for oscillation parameters fixed at their best fit points and $s_{13}=0, \alpha_{32}=\pi$, and $\alpha_{12}=0, \pi / 4, \pi / 2,3 \pi / 4, \pi$.

LFV ones. Events with $\tau$ are more difficult to reconstruct, so in our analysis we only consider events where at most one of the four leptons is a $\tau$.

Our observables depend only on the neutrino mass matrix, Eq.(1.2). We parametrise it in terms of the PMNS matrix: $M=U \operatorname{diag}\left(m_{1}, m_{2}, m_{3}\right) U^{T}$. We use as input parameters the two squared mass differences, and the mixing angles $\theta_{12}$ and $\theta_{23}$. The parameters of our analysis are: the lightest neutrino mass $m_{0} ; s_{13}=\sin \theta_{13}$; the Dirac phase $\delta$; and the two physical Majorana phases $\alpha_{12}$ and $\alpha_{32}$, defined as $\alpha_{i j}=\alpha_{i}-\alpha_{j}$ with $\alpha_{i}$ the phase associated to the $i$ mass eigenstate.

In Fig. (1) we show the branching ratio into each channel as a function of the lightest neutrino mass. Colour red corresponds to normal hierarchy (NH) and blue to inverted hierarchy (IH). The solid line is for $s_{13}=0$ and the dashed line for $s_{13}=0.1$. For fixed $m_{0}$, the allowed interval arises due to the dependence on the phases and the uncertainty in neutrino parameters. The wide allowed region for IH and quasi degenerate (QD) neutrinos (in the limit of large values for $m_{0}$ ) suggest a strong dependence on the phases.

The allowed regions for $\mathrm{NH}$ and $\mathrm{IH}$ in the ee channel are very well separated because in the case of NH this channel is suppressed. The reason is that, contrary to the case of IH and QD, for $\mathrm{NH}$ in the limit of vanishing $m_{0}, B R_{e e}$ is suppressed by $r=\Delta m_{21}^{2} /\left|\Delta m_{31}^{2}\right|$ or by powers of $s_{13}$ :

$$
\begin{aligned}
\mathrm{BR}_{e e}^{\mathrm{NH}, m_{0}=0} & \approx s_{12}^{4} r+2 s_{12}^{2} s_{13}^{2} \sqrt{r} \cos \left(\alpha_{32}-2 \delta\right), \\
\mathrm{BR}_{e e}^{\mathrm{IH}, m_{0}=0} & =\frac{1}{2}\left(1-\sin ^{2} 2 \theta_{12} \sin ^{2} \frac{\alpha_{12}}{2}\right)=\frac{3}{2} \mathrm{BR}_{e e}^{\mathrm{QD}},
\end{aligned}
$$

Therefore, seeing a suppression in the channel to two electrons would be a clear signature of a hierarchical spectrum with normal order. 

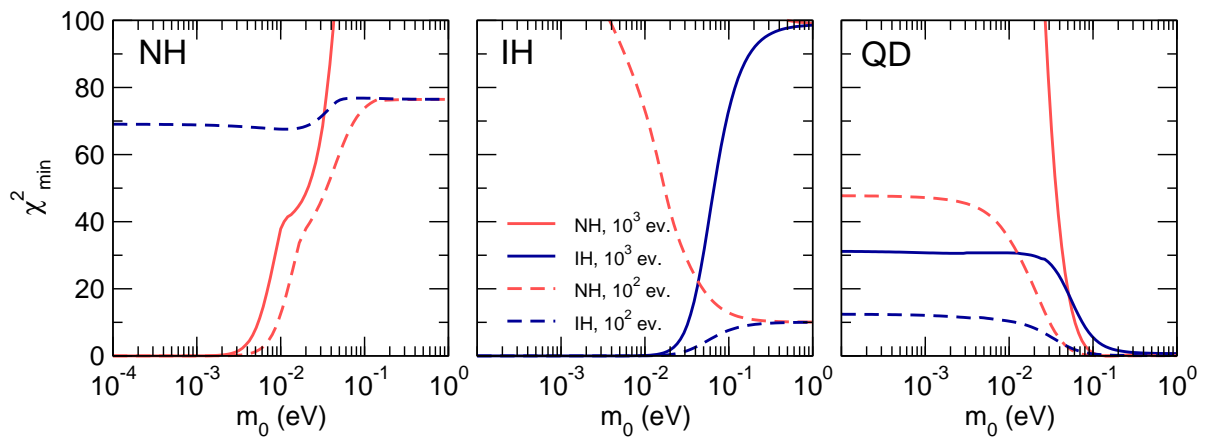

Figure 2: $\chi_{\min }^{2}$ vs $m_{0}$ assuming a true NH (left), IH (middle), and QD spectrum (right). The $\chi^{2}$ is shown for $10^{2}$ (dashed) and $10^{3}$ (solid) events. In the fit we assume either NH (light-red) or IH (dark-blue). We adopt the following true parameter values. Left: $m_{0}=0, \mathrm{NH}, \alpha_{32}=\pi$; middle: $m_{0}=0, \mathrm{IH}, \alpha_{12}=0$; right: $m_{0}=0.15 \mathrm{eV}, \alpha_{12}=0.1 \pi, \alpha_{32}=1.6 \pi ;$ and always $s_{13}=0$.
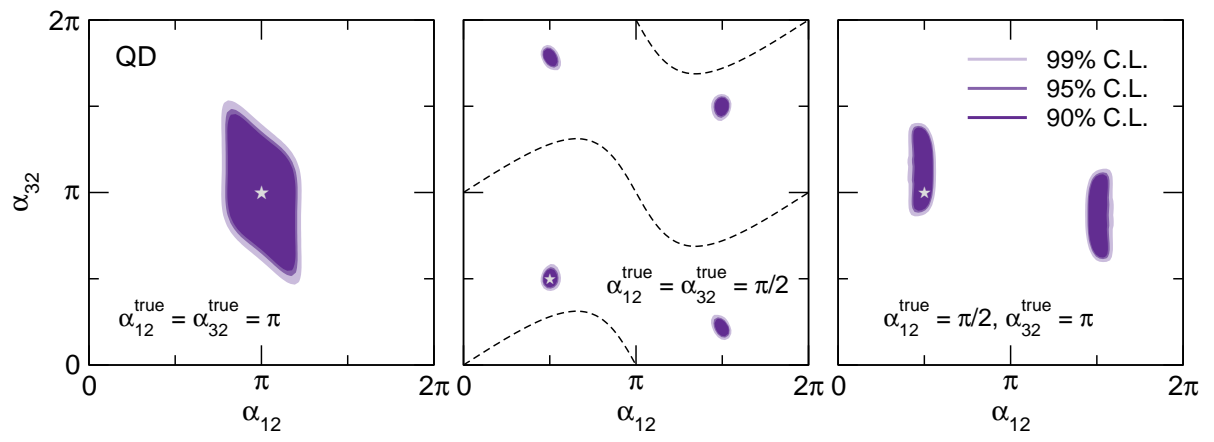

Figure 3: Determination of the Majorana phases for QD spectrum $\left(m_{0}=0.15 \mathrm{eV}\right)$ from $10^{3}$ events. We assume $s_{13}^{\text {true }}=0$ and three example points for the true values of the Majorana phases. The dashed lines in the middle panel correspond to the true values of the phases for which the degenerate solution appears at a CP conserving value of $\alpha_{32}$.

\subsection{Determination of the neutrino mass spectrum}

In Fig.(2) we try to quantify the ability of the data to determine the neutrino mass spectrum by performing a $\chi^{2}$ analysis. We assume some true values for the parameters, which are specified in the caption. Then these data are fitted with both possibilities of $\mathrm{NH}$ (red) and IH (blue), and a given value of $m_{0}$. We show the results for $10^{3}$ and $10^{2}$ events. A true NH (left panel) can be identified with high significance, due to the distinctive signature provided by $B R_{e e}$. A true IH or QD spectrum (middle and right panels) are more difficult to identify, but good sensitivity is obtained depending on the number of events. We have found that it is possible to decide whether the lightest neutrino mass is smaller or larger than roughly $0.01 \mathrm{eV}$, which marks the transition between hierarchical and quasi-degenerate spectra. If it is smaller the mass ordering (normal vs inverted) can be identified.

\subsection{Determination of Majorana phases}

We have also explored the possibility of measuring $\mathrm{CP}$ phases within this neutrino mass model at LHC. There is no explicit CP violation in the decay of the doubly charged scalar, but it depends in a CP conserving way on the Majorana phases. In Fig.(3) we assume a QD spectrum and some 

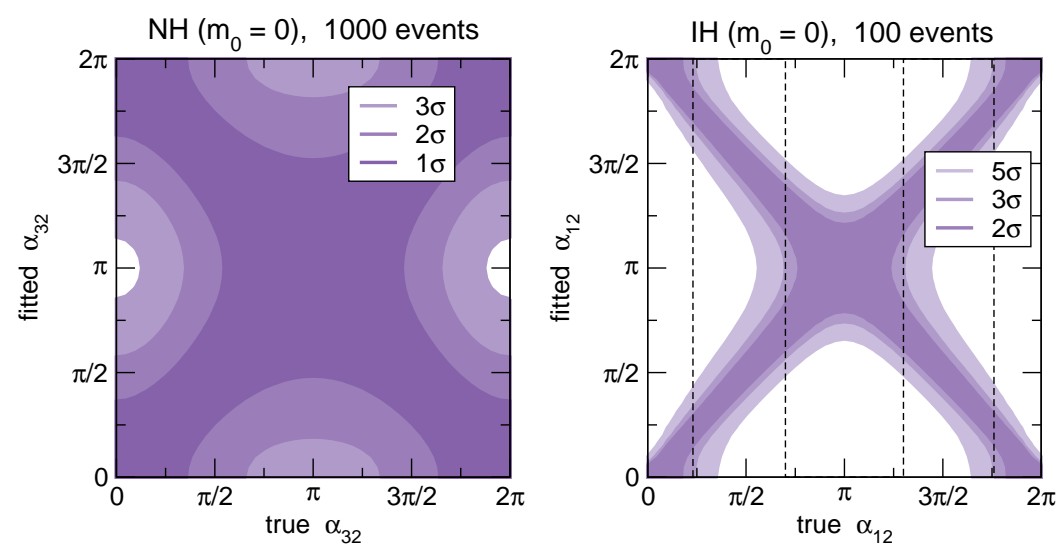

Figure 4: Determination of the Majorana phase for vanishing lightest neutrino mass. We assume $s_{13}^{\text {true }}=0$. Left: $1,2,3 \sigma$ ranges for $\alpha_{32}$ as a function of its true value for $\mathrm{NH}$ assuming $10^{3}$ events. Right: $2,3,5 \sigma$ ranges for $\alpha_{12}$ as a function of its true value for IH assuming $10^{2}$ events. The dashed vertical lines indicate the region where $\mathrm{CP}$ violating values of $\alpha_{12}$ can be established at $3 \sigma$.

true values for the Majorana phases. We find that the accuracy to reconstruct the value of the true phases depends on the true values that we assume. As can be seen from the plots, some degenerate solutions appear. There are two kinds of degeneracies. First, as a consequence of CP invariance, the branching ratios are invariant under the simultaneous change of $\alpha_{i j} \rightarrow 2 \pi-\alpha_{i j}, \delta \rightarrow 2 \pi-\delta$. The second degeneracy appears due to the fact that the phases $\alpha_{12}$ and $\alpha_{32}$ always appear in the same particular combination in the limit $s_{13}=0$.

In Fig.(4) we show the allowed regions for the physical phase as a function of the true value for the phase. In the case of $\mathrm{NH}$ the sensitivity to $\alpha_{32}$ is very bad, even if we consider $10^{3}$ events. In the case of $\mathrm{IH}$, the determination is very efficient already with $10^{2}$ events, apart from the degeneracy $\alpha_{12} \rightarrow 2 \pi-\alpha_{12}$.

\section{Conclusions}

A Higgs triplet in the $\mathrm{TeV}$ scale offers an appealing mechanism to provide mass to neutrinos which can be directly tested at the LHC. Such a scenario opens the possibility to measure the Majorana phases of the lepton mixing matrix, which in general is a very difficult task.

The observation of a doubly charged scalar at LHC would be a great discovery of physics beyond the Standard Model, but it does not confirm the Higgs triplet mechanism for neutrino masses, since doubly charged particles decaying into leptons are predicted in many models. In case such a particle is indeed found at LHC, various consistency checks will have to be performed.

\section{References}

[1] G. B. Gelmini and M. Roncadelli, Phys. Lett. B 99 (1981) 411.

[2] A. Hektor, M. Kadastik, M. Muntel, M. Raidal and L. Rebane, Nucl. Phys. B 787, 198 (2007). T. Han, B. Mukhopadhyaya, Z. Si and K. Wang, Phys. Rev. D 76, 075013 (2007).

[3] J. Garayoa and T. Schwetz, JHEP 0803 (2008) 009. 